\title{
Lead isotopic measurements in archeological objects
}

\author{
Igor M. Villa
}

Received: 31 March 2009/Accepted: 22 July 2009 /Published online: 13 August 2009

(C) Springer-Verlag 2009

\begin{abstract}
Pb}$ isotopic analyses on metal artifacts help constrain their provenance. Useful and reliable data require analytical protocols that ensure accuracy and monitor precision. The interpretation can sometimes be ambiguous because natural ore deposits frequently have overlapping $\mathrm{Pb}$ isotopic compositions. In most cases, however, provenances can be reliably narrowed down. For the Alpine region, a pattern of small, strongly localized mine exploitations in pre-Roman times, supplemented by some longdistance trading, is taking shape.
\end{abstract}

Keywords Lead $\cdot$ Isotopic measurements .

Archeological objects · Natural ore deposits · Provenance

\section{Introduction}

The provenance of metal artifacts in the early history of metallurgy has been a major question in archeology for many decades. Most metal ores contain a large and variable number of base metals, as major and minor elements. Therefore, it would seem straightforward to use these contaminant elements as indicators of the major element provenance. However, metallurgical improvements have led to a progressively more conscious control of "impurity"

\section{M. Villa $(\square)$}

Institut für Geologie, Universität Bern,

Baltzerstrasse 3,

3012 Bern, Switzerland

e-mail: igor@geo.unibe.ch

\section{M. Villa}

Dipartimento di Scienze Geologiche e Geotecnologie,

Università di Milano Bicocca,

20126 Milan, Italy concentrations. Impurities can be reduced to practically zero (an extreme example is modern electrolytical purification), but they can also be selectively admixed (e.g., Sn and As to $\mathrm{Cu}$ ) to obtain an alloy with desirable properties. Therefore, minor and trace element concentrations have an inherent ambiguity, as their abundances in the cultural artifact no longer represent the original abundances of the ore deposit.

Many metal ores and most metal artifacts in preindustrial cultures (copper, tin, silver, gold, and even iron), contain traces of $\mathrm{Pb}$, which has a unique characteristic among base metals: its isotopic composition is not constant, but varies from one ore deposit to the other. Contrary to element concentrations, isotopic ratios are not modified by depletion: even if most $\mathrm{Pb}$ has been removed from the $\mathrm{Sn}$ used for bronze alloys, the minute remaining quantities preserve the isotopic composition of the source ore body. Therefore, $\mathrm{Pb}$ isotopic ratios have been used for a long time to identify the mining region from which the ore was derived (Grögler et al. 1966). Each mining district that shares a common geological history also shares a common $\mathrm{Pb}$ isotopic "fingerprint."

To keep a complex argument simple, we can note that, among the four stable isotopes of $\mathrm{Pb}$, three are radiogenic daughter products of radioactive decay: ${ }^{208} \mathrm{~Pb}$ is produced by the decay of ${ }^{232} \mathrm{Th},{ }^{207} \mathrm{~Pb}$ by that of ${ }^{235} \mathrm{U}$, and ${ }^{206} \mathrm{~Pb}$ by that of ${ }^{238} \mathrm{U}$. ${ }^{204} \mathrm{~Pb}$ is not the product of any radioactive decay and its amount has not changed. Because radioactive decay is irreversible; as a rule, terrestrial rocks have $\mathrm{Pb}$ isotope ratios $\left({ }^{208} \mathrm{~Pb} /{ }^{204} \mathrm{~Pb},{ }^{207} \mathrm{~Pb} /{ }^{204} \mathrm{~Pb}\right.$, and $\left.{ }^{206} \mathrm{~Pb} /{ }^{204} \mathrm{~Pb}\right)$ that increase monotonically with time. The one exception are sulfide ores. Uranium is not incorporated in sulfides (galena, fahlore, etc.), so that, in these particular ores, the $\mathrm{Pb}$ isotopic composition is frozen at the time of the oreforming event, when $U$ and Th removal stopped adding 
radiogenic $\mathrm{Pb}$ daughter isotopes. This ensures that, once a sulfide ore is formed, it will retain a constant $\mathrm{Pb}$ isotopic composition, i.e., gains an individual $\mathrm{Pb}$ fingerprint. A much more detailed discussion of $\mathrm{Pb}$ isotopic evolution is presented in the textbook by Tolstikhin and Kramers (2008).

While $\mathrm{Pb}$ isotopic fingerprinting potentially could identify the provenance of an archeological object, there are a number of related difficulties, which will be discussed in the present contribution.

Because the geological history of a certain geographic area (e.g., the Mediterranean) normally consists of discrete periods of geological activity, it is a common observation that ore-forming processes tend to be clustered in space ("mining districts") and time ("ore-forming events"). This ensures that the $\mathrm{Pb}$ isotopic fingerprints of related ore bodies will be similar to each other and, by far, not as unique as archeometrists would like them to be. As an example, let us consider the Aegean area: over a fairly large region, the genesis of the ores occurred in the geologically recent past, during the last 20 million years; mining activity has exploited many different ore bodies, which all have a similar fingerprint (sometimes identical, sometimes with small differences). From the historical-political point of view, however, it makes a significant difference to be able to discriminate whether one particular trade route with one particular island was active or inactive, for example, due to alliances, colonization, or hostilities. The task for the archeometrist is thus to resolve ambiguities and nonunicities as much as possible.

The first way to reduce the overlap between the measured values is by improving the analytical precision. Two values are said to be statistically indistinguishable when their difference is smaller than the sum of the analytical uncertainties with which they were measured. Thus, it may be impossible to tell apart two values if the measurement precision is low, but it may be possible to resolve them if the precision increases. A simple analogy is photographic resolution of faces in a crowd with wideangle or telephoto lenses.

Precision alone is only part of the solution. On one hand, precision is expected a priori to be proportional to $\sqrt{\mathrm{N}}$, the square root of all ions that hit the collector device in the measuring instrument, i.e., to sample size. For some samples, size is no concern; sewer lead pipes in Pompeii contain enough $\mathrm{Pb}$ for billions of analyses. For other samples (e.g., gold coins), museum curators are unlikely to accept the destruction of grams of material for just one $\mathrm{Pb}$ analysis. In general, archeometric analyses often involve a trade-off between achieving an acceptable precision and destroying an acceptable mass of sample. A decisive criterion that data must fulfill is accuracy. As will be made clearer in the following paragraphs, it is not always obvious that the ions counted by the analytical equipment are the same that were frozen in by the ore.

The second way to reduce ambiguities is to enlarge the context with other indicators. As was mentioned above, trace element concentrations vary as metallurgy evolves; however, because of similarities in the chemical behavior of element groups that are related in the periodic system, the metallurgical processing affects certain element ratios much less than the individual element concentrations. Thus, by a judicious investigation of the multielement abundance patterns, some characteristics that discriminate among different ore bodies can be retraced in the metallic artifacts (cf., Pernicka 1999; see also, Artioli et al. 2008).

\section{Errors}

The distinction between random errors and systematic errors, i.e., between precision and accuracy, is well established in science, but is not always as clear as it should to users who were educated in other disciplines. In essence, an experimental determination of a physical quantity is always a gamble between the experimenter, who tries to elicit a numerical response from an apparatus aimed at an object, and the laws of physics, which have the unpleasant, albeit impersonal, tendency of hiding complexity unknown to the experimenter. In very crude terms, "accuracy" means that the measured value is right within its uncertainty. Its opposite, inaccuracy, is caused by a systematic error, the systematic deviation of the measured value from the "true" one. The recognition of inaccuracies is impossible by simple repetition of the same analytical protocol (unless the source of systematic error is increasing or decreasing with time) and requires comparisons of different kinds of analyses, as well as analyses of commonly accepted interlaboratory standards.

On the other hand, precision reflects the reproducibility of a given measurement: given a large number of repeat experiments, the spread of the results around their average should be totally random and the magnitude of the spread will reflect the precision. As an example, consider the task of weighing a liquid. If the liquid is stored in a closed container of known weight, one needs to weigh the empty container several times, then weigh the filled container, determining the respective averages and dispersions; the average value and uncertainty of the liquid weight are obtained by subtraction and by quadratic compounding, respectively. If the liquid is stored in an open container, one may repeat the same operations and get different weights on different days-however, the data spread is no longer a random effect because evaporation causes a systematic error. Therefore, in this case, the dispersion of the results must not be taken to reflect the precision. 


\section{Analytical techniques}

The choice of the analytical protocol to be used is sometimes considered to be subject to the whims of fashion: analyses are then labeled "modern" or "traditional." In actual fact, there is no such distinction and the only point that can and must be considered is the extent to which an analysis is accurate and its analytical uncertainty.

In order to perform a $\mathrm{Pb}$ isotopic analysis, the $\mathrm{Pb}$ must be brought from the sample into the mass spectrometer and be ionized. It sounds trivial, but is not, that only $\mathrm{Pb}$ from the sample, possibly all of it, and certainly nothing else, should be analyzed.

Most ore minerals and metal artifacts are soluble in aqua regia. Therefore, dissolution by aqua regia is the method of choice when doing bulk analyses. One potential exception, tin-rich samples, might produce a $\mathrm{SnO}_{2}$ precipitate if chloride formation was incomplete. This was not observed for acid/sample ratios exceeding 1,000. Moreover, it is thought to be a minor problem, as $\mathrm{PbCl}_{2}$ stays in solution even if a precipitate is formed.

A complementary technique uses spatially resolved analyses in which selected spots of a sample are vaporized by a laser connected to a plasma mass spectrometer (in which case, the following description of the chemical purification is not applicable).

The dissolved sample contains small (or large) amounts of $\mathrm{Pb}$ mixed with all other elements contained before dissolution. It is necessary to chemically remove all these interfering elements, as experience directly shows that the measurement accuracy depends on $\mathrm{Pb}$ purity. This is normally done with cation or anion exchange resins, which retain or release specific elements with specific acids of specific concentration. In the Bern laboratory, $\mathrm{Pb}$ is separated from other metals with the $\mathrm{Sr} \bullet \mathrm{Spec}^{\mathrm{TM}}$ resin (EIChroM Industries) described by Horwitz et al. (1992). Its use for $\mathrm{Pb}$ was reported by Gale (1996). Because that paper contains a number of errors, the corrected procedure is detailed here. About $100 \mu \mathrm{L}$ of $\mathrm{Sr} \bullet \mathrm{Spec}^{\mathrm{TM}}$ resin are filled in a $3-\mathrm{mm}$ diameter hand-made PTFE column. The height/width ratio is approximately 4. The sample solution is loaded in $0.5 \mathrm{~mL} 1 \mathrm{M} \mathrm{HNO}_{3}, 1.5 \mathrm{~mL}$ of which is also used to wash out the matrix metals, while $\mathrm{Pb}$ is very strongly retained on the resin. $\mathrm{Pb}$ is then eluted with $3 \mathrm{~mL} 0.01 \mathrm{M} \mathrm{HNO}_{3}$ and is ready for analysis.

Use of a laser has the great advantage of allowing a reduction of the analyzed sample size. This reduction comes at a price, as the small total number of counted ions entails a loss of precision (see above). Moreover, one important cause of concern that needs to be examined on a case-by-case basis is the presence of interfering ions. Because no chemical separation can be performed on the laser evaporates, elements such as $\mathrm{Hg}$ (which also has a ${ }^{204} \mathrm{Hg}$ isotope) are analyzed at the same time as $\mathrm{Pb}$.
Mercury can be corrected by measuring the ${ }^{202} \mathrm{Hg}$ isotope and assuming a constant $\mathrm{Hg}$ isotopic ratio; this may or may not be an accurate assumption, and as a rule, it is prudent to avoid large corrections lest a small inaccuracy in the amount or isotopic composition of $\mathrm{Hg}$ is amplified and produces a large systematic inaccuracy on the ${ }^{204} \mathrm{~Pb}$.

The choice of mass spectrometer on which to perform the analysis is essentially limited to two designs: thermal ionization mass spectrometer (TIMS) and plasma ionization multicollector mass spectrometer (PIMMS). In the TIMS, ionization is achieved by a hot filament on which the sample solution is deposited. Each element is evaporated and ionized at a characteristic filament temperature. This allows selective "burning off" of interfering elements such as $\mathrm{Hg}$, which are more volatile than $\mathrm{Pb}$. The largest source of uncertainty is the isotope fractionation during the analysis because it cannot be monitored on a $\mathrm{Pb}$ isotope pair known to have a fixed natural ratio. In the PIMMS, the sample solution is ionized by introducing it into a 9,000-K plasma. All elements are ionized simultaneously, and this has important analytical implications. Mass fractionation can be monitored by adding a small quantity of a tracer element to the element to be analyzed. In a $\mathrm{Pb}$ analysis, it is usual to add a few nanograms of $\mathrm{Tl}$. The reasoning, accepted by the overwhelming majority of research groups after extensive tests, is that the ${ }^{203} \mathrm{Tl} /{ }^{205} \mathrm{Tl}$ ratio is a known constant; because $\mathrm{Tl}$ and $\mathrm{Pb}$ are ionized together and fractionated by the same mechanism and because neither mass 203 nor mass 205 interfere with a $\mathrm{Pb}$ isotope, the fractionation of the $\mathrm{Tl}$ isotopic ratio provides a rapid and accurate correction for the $\mathrm{Pb}$ fractionation as well. This ensures about 10-50 times smaller fractionation-derived analytical uncertainties than TIMS analyses. However, a drawback of the simultaneous plasma ionization is that whatever impurities have not been removed by the chemical separation can interfere with the analysis. Such effects can be either direct isobaric interference (such as ${ }^{204} \mathrm{Hg},{ }^{188} \mathrm{Os}^{16} \mathrm{O}^{+}$, etc. with ${ }^{204} \mathrm{~Pb}$ ) or a much more subtle artifact whereby the focusing of the ion beam in the source is modified by extraneous ions having multiple oxidation states, such as $\mathrm{Fe}^{2+} / \mathrm{Fe}^{3+}$, resulting in irreproducible analytical conditions. Preventing this requires a very thorough chemical purification procedure of $\mathrm{Pb}$ from all other elements. Finally, single-collector plasma ionization mass spectrometers are certain to suffer from systematic biases, such as plasma instabilities and low intensities, which make them unsuitable for accurate $\mathrm{Pb}$ isotope work.

\section{Interpretive principles}

It is important to reiterate the concept that $\mathrm{Pb}$ isotope analyses may provide an "age," but that this age is in no 
case the age of the artifact. The ${ }^{207} \mathrm{~Pb} /{ }^{206} \mathrm{~Pb},{ }^{207} \mathrm{~Pb} /{ }^{204} \mathrm{~Pb}$, and ${ }^{206} \mathrm{~Pb} /{ }^{204} \mathrm{~Pb}$ ratios only can indicate the geological age of the deposit.

It is necessary to address only archeological questions that are relevant to the potential of $\mathrm{Pb}$ isotope systematics. Mankind has exploited thousands of ore deposits in the last 5,000 years, and the unique identification of sources is not always possible. In order to maximize the fingerprinting information provided by the $\mathrm{Pb}$ isotopic signature, it is most useful to maximize data exploitation.

The four isotopes of $\mathrm{Pb}$ can be combined into 24 different isotope ratios, at most three of which are truly independent. As an example, the triplet $\left({ }^{207} \mathrm{~Pb} /{ }^{206} \mathrm{~Pb}\right.$, ${ }^{207} \mathrm{~Pb} /{ }^{204} \mathrm{~Pb},{ }^{204} \mathrm{~Pb} /{ }^{208} \mathrm{~Pb}$ ) contains three independent ratios, while $\left({ }^{207} \mathrm{~Pb} /{ }^{206} \mathrm{~Pb},{ }^{207} \mathrm{~Pb} /{ }^{204} \mathrm{~Pb},{ }^{204} \mathrm{~Pb} /{ }^{206} \mathrm{~Pb}\right)$ only contains two. Each sample can be plotted in a three-dimensional graph whose axes are the three chosen independent ratios. Among all possible triplets of independent isotopic ratios, the best candidates for the three axes are triplets of common-denominator ratios because only in that case do mixtures between samples lie on a straight tie line between the samples' points (cf., Villa 2001). As visualization is most simple in two dimensions, it is necessary to recall that any projection is associated with loss of information. This means that at least two projections are needed to fully display the information contained in the three independent ratios (typically, an $x-y$ and an $x-z$ projection).

In the mid-1960s, when the first archeological objects were analyzed (e.g., Grögler et al. 1966), analytical protocols were less optimized than those used today. The most critical point of both precision and accuracy was the measurement of ${ }^{204} \mathrm{~Pb}$, and this led some archeologists to forgo the information provided by the latter isotope and limit the discussion to just three isotopes, i.e., two independent ratios (usually ${ }^{207} \mathrm{~Pb} /{ }^{206} \mathrm{~Pb}$ and ${ }^{208} \mathrm{~Pb} /{ }^{206} \mathrm{~Pb}$ ). From what has been said above, it is clear that such a reduction to a two-dimensional correlation diagram entails a loss of information and greater ambiguity in the discrimination of provenances.

\section{Examples}

Clearly, a metal object can possibly result from artificial mixing of ores with heterogeneous provenances, e.g., by remelting of scrap metals. This was already documented by Grögler et al. (1966). It is the archeologists' task to identify objects with a high likelihood of reflecting a single source (jewelry is mostly produced from a small metal batch and, once acquired, is passed on as family heirloom without remelting) from those with a low one (such as the thousands of lead pipes of the Pompeii sewer system; see,
Boni et al. 2000). As the goal of archeometric research is recognizing a context, archeological criteria should drive the selection of samples to be analyzed.

The trade relations and political alliances of the Samnites during the fourth century $\mathrm{BC}$ were not constrained with certainty from available historical information. Silver and pewter jewelry found in the Saticula necropolis (Balassone et al. 2002) demonstrates that Attica, and neither Carthago nor Etruria, was the Samnites' supplier of silver and pewter at that time.

The question of Late Neolithic trade routes was addressed in the Ph.D. thesis of Cattin (2008), a chapter of which is presented in this volume (Cattin et al. 2009). One of the most surprising findings was that, in the Late Neolithic (and even more so in the Bronze Age), metal objects have a great variety of provenances and that some of the isotopic fingerprints do not coincide with any major ore deposit known today. This can only be explained by exploitation of small and smallest ore bodies, resolvably distinct from (i.e., geological similar to, but different from) the major ones known from historical times.

The Valais region in Switzerland hosts dozens of historically recorded small and very small ore deposits. In her Ph.D. thesis, Guénette-Beck (2005) charted the isotopic compositions of Valais ore minerals and compared them to metal objects from pre-Roman to Medieval times. The isotopic fingerprints of the Valais ores were observed to be, as expected, clustered around values typical of Late Paleozoic-Early Mesozoic mineralization events. This made them similar to the fingerprints of many other ore bodies of similar age, scattered all over a belt ranging from Spain to Bohemia and the Balkans. Differences between individual Valaisan ore bodies were mostly greater than the analytical uncertainties, i.e., were resolvable. The analyzed objects were remarkable, in that most were clearly compatible with a local origin of ores. While most of the identifications were not rigorously unique, it is tempting to resort to Occam's razor and hypothesize that, if a metal object is found near an ore body that matches isotopically, it is more likely that the ore was of local origin than imported from far away.

This, and many more ongoing, unpublished studies bring mounting evidence of local exploitation of small, local sources. Metallurgy appears to have been a diffuse knowledge, rather than the monopoly of a few large and specialized exporting centers. This brings one complication: instead of the half dozen "large" ore sources considered by Grögler et al. (1966) in the Mediterranean and Central Europe, we now must study and characterize several thousands only in Northern Italy. An effect of this "crowding" of sources is that a given $\mathrm{Pb}$ isotopic fingerprint is becoming less and less unambiguous. 


\section{In lieu of a conclusion}

In this methodological paper, I have tried to point out what must be taken into account when doing an archeometric $\mathrm{Pb}$ isotope analysis. Rigor is timeless, and analytical work must always be performed in such a way that it shall never be a question whether or not the data are accurate and reliable. Calibrations should account for a significant part of the total time spent doing analyses.

If you have read this far, then now it is time to go out and get new data.

Acknowledgements I am grateful to Y. Bassiakos, A.L. Burke, F. Cattin, I. Giunti, and one unnamed referee for their comments.

\section{References}

Artioli G, Baumgarten B, Marelli M, Giussani B, Recchia S, Nimis P, Giunti I, Angelini I, Omenetto P (2008) Chemical and isotopic tracers in alpine copper deposits: geochemical links between mines and metal. Geo Alp 5:139-148

Balassone G, Boni M, Di Maio G, Fariello M, Villa IM (2002) Analysis of the rings found in the Samnitic necropolis of Saticula (Sant'Agata dei Goti, Benevento, Italy). In: Jerem E, Biró KT (eds) Proceedings of the 31 st symposium on archeometry. Archeopress, Oxford, pp 279-283

Boni M, Di Maio G, Frei R, Villa IM (2000) Lead isotopic evidence for a mixed provenance of Roman water pipes from Pompeii. Archeometry 42:201-208

Cattin F (2008) Modalités d'approvisionnement et modalités de consommation du cuivre dans les Alpes au $3^{\mathrm{e}}$ millénaire avant notre ère. Ph.D. thesis, Genève

Cattin F, Villa IM, Besse M (2009) Copper supply during the Final Neolithic of the Saint-Blaise/Bains des Dames site (Neuchâtel, Switzerland). This volume

Gale NH (1996) A new method for extracting and purifying lead from difficult matrices for isotopic analysis. Anal Chim Acta 332:15-21

Grögler N, Geiss J, Grünenfelder M, Houtermans FC (1966) Isotopenuntersuchungen zur Bestimmung der Herkunft römischer Bleirohre und Bleibarren. Z Naturforsch 21:1167-1172

Guénette-Beck B (2005) Minérais, métaux, isotopes: recherches archéométriques sur les mines de plomb et d'argent en Valais, Suisse. Ph.D. thesis, Lausanne

Horwitz EP, Chiarizia R, Dietz ML (1992) A novel strontium-selective extraction chromatographic resin. Solv Extr Ion Exch 10:313-336

Pernicka E (1999) Trace element fingerprinting of ancient copper: a guide to technology or provenance? In: Young SMM, Pollard AM, Budd P, Ixer RA (eds) Metals in antiquity. BAR International Series 792. Hadrian Books, Oxford, pp 163-171

Tolstikhin IN, Kramers JD (2008) The evolution of matter. Cambridge University Press, Cambridge

Villa IM (2001) Radiogenic isotopes in fluid inclusions. Lithos $55: 115-124$ 